\title{
An Explicit Formula Based Estimation Method for Distribution Network Reliability
}

\author{
Yue Xiang, Member IEEE, Yunche Su, Yang Wang, Junyong Liu, Member IEEE, Xin Zhang, Member IEEE
}

\begin{abstract}
An improved explicit estimation algorithm is proposed for reliability estimation of the distribution network. Firstly, hierarchical clustering is used to identify and cluster typical feeders based on the topology structure. Secondly, the explicit formula of reliability index under each typical feeder topology is derived by regression analysis, to establish the model for network reliability estimation. Numerical simulations show the suitability of the proposed method in obtaining accurate reliability index for diversified network topology.
\end{abstract}

Index Terms-reliability evaluation, explicit estimation, hierarchical clustering, distribution network planning

\section{INTRODUCTION}

$\mathbf{T}$ HE system average interruption frequency index (SAIFI) and system average duration index (SAIDI) [1] are widely applied as reliability indicators for power outages in distribution networks.

$$
\begin{gathered}
S_{F}=\sum_{k=1}^{N_{E}} N_{E k} \lambda_{k}\left(\beta_{A k} u_{A}+\beta_{B k} u_{B}+\beta_{C k} u_{C k}\right) \\
S_{D}=\sum_{k=1}^{N_{E}} N_{E k} \lambda_{k}\left(\beta_{A k} T_{A}+\beta_{B k} T_{B}+\beta_{C k} T_{C k}\right)
\end{gathered}
$$

where $S_{F}$ is SAIFI, $S_{D}$ is SAIDI, $\lambda_{k}$ is the failure rate of the $k_{t h}$ type of equipment, $N_{E}$ is the equipment amounts, $\beta$ is the affected customers after a failure caused by $k_{\text {th }}$ type equipment. $T_{A}, T_{B}$, and $T_{C}$ represent the corresponding outage time from Table $\mathrm{A}$ in [2]. $u_{A}, u_{B}$, and $u_{C}$ are $0-1$ variables which reflect the status of power failures respectively. In (12), $\beta$ is hard to calculate due to the complexity of network topology and load distribution.

The SAIFI and SAIDI are often embedded into the optimization model for distribution network planning. However, the quantitative evaluation of such index is often dominated by analytical or simulation method [3]. Either method requires to search and analyze the network topology for $\beta$ calculation. Consequently, the optimization of following network planning schemes is greatly restricted. In addition, the estimation algorithm in [4] proposed a simplified method to estimate $\beta$. However, there were

This work was supported by National Natural Science Foundation of China (51807127), the Sichuan Science and Technology Program (2019YFH0171) and International Visiting Program for Excellent Young Scholars of Sichuan University. Y. Su and Y. Wang are the corresponding authors.

Y. Xiang, Y. Wang, J. Liu are with College of Electrical Engineering, Sichuan University, Chengdu 610065, China (e-mail: xiang@scu.edu.cn, yangwang@stu.scu.edu.cn, liujy@scu.edu.cn).

Y. Su is with the Planning and Evaluation Center, State Grid Sichuan Economic Research Institute, Chengdu 610041, China (e-mail: yunchesu@gmail.com).

X. Zhang is with Energy and Power Theme, School of Water, Energy and Environment, Cranfield University, Cranfield, MK43 0AL, UK (e-mail: xin.sam.zhang@gmail.com). significant assumptions and empirical judgment in the estimation algorithm which still needs to be improved.

An improved estimation algorithm by using explicit formula is proposed to obtain the reliability index for distribution network planning. The typical feeder structures are classified and clustered by hierarchical clustering algorithm based on the tree edit distance (TED). Reliability index function is derived from regression analysis by considering the fault isolation and load transfer. The proposed algorithm considers the various impacts of network topology and system faults, and offers an embedded optimization model for distribution network planning.

\section{Hierarchical Clustering of Network Topology}

\section{A. Topological characterization of feeder structure}

The analysis of feeder topology is based on fault isolation and load transfer. Therefore, the nodal location with branch structure is extracted from the distribution network, while the detailed assets are usually ignored. One specific topology diagram can be seen from Fig. 1 .

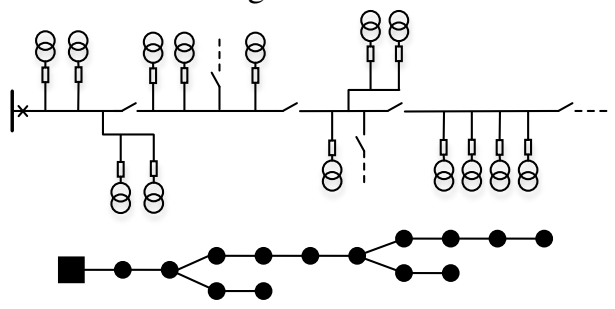

Fig. 1. Topology characterization of feeders

\section{B. Similarity analysis based on TED}

For network topology clustering, it is necessary to measure the distance between two different topologies. Therefore, TED [5] is used to measure the similarity between different networks. TED indicates the minimum cost from one tree to another.

$$
D\left(M_{1}, M_{2}\right)=\min \sum_{i=1}^{|S|} C\left(s_{i}\right)
$$

where $\mathrm{S}=\left\{s_{1}, s_{2}, \ldots, s_{k}\right\}$ denotes a set of tree edit operations converted from $\mathrm{M}_{1}$ to $\mathrm{M}_{2},|S|$ is the number of tree edit operations in $S$ set, and $\mathrm{C}\left(s_{i}\right)$ is the cost of the $s_{i}$-step operation.
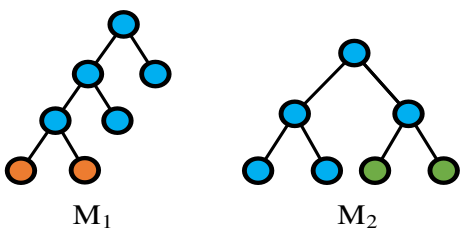

Fig. 2. Identification of TED in different tree structures 
The strategy of TED for computing trees is to decompose the tree into multiple subtrees, and calculate the TED between subtrees from bottom to the top until the TED between the two original trees is completed.

Fig. 2 shows two tree structures $\mathrm{M}_{1}$ and $\mathrm{M}_{2}$, assuming the nodes in $\mathrm{M}_{1}, \mathrm{M}_{2}$ are numbered according to the inorder traversal. In addition, the isomorphic set $\mathrm{G}\left(\mathrm{M}_{1}, \mathrm{M}_{2}\right)$ is defined. The $\mathrm{G}$ is composed of $(i, j)$, which represent the node number in $\mathrm{M}_{1}$, and $\mathrm{M}_{2}$ respectively. From Fig.2, the blue nodes represent the isomorphic set of two trees. When the nodes that do not belong to $\mathrm{G}$ in the two trees are deleted, the two trees will get the same substructure. Therefore, the following formula can be used to calculate the cost from $\mathrm{M}_{1}$ to $\mathrm{M}_{2}$.

$$
D\left(\mathrm{M}_{1}, \mathrm{M}_{2}\right)=\min \left(\sum_{(i, j) \in \mathrm{M}} C_{c}(i, j)+\sum_{i \notin \mathrm{M}} C_{d}(i)+\sum_{j \notin \mathrm{M}} C_{i}(j)\right)
$$

where $C_{c}, C_{d}$, and $C_{i}$ are the costs of node change, node deletion and node insertion respectively. To avoid size impact on similarity analysis, the tree topology is adjusted to have the same number of nodes for TED calculation.

\section{Hierarchical clustering for feeder structure}

Although the purpose of TED algorithm is to calculate the degree of difference between feeder topology, there is no absolute attribute value for each feeder topology. In this paper, the agglomerative hierarchical clustering algorithm based on distance is adopted to cluster the typical topologies $K$. Firstly, the distance between $N$ feeders in the region is calculated, then the two nearest feeders are merged to form $N-1$ classes. The distance between $N-1$ feeders is further calculated, and the above process is repeated until all feeders are merged into one class or the termination condition is met. In the clustering process, TED is used as the distance between feeder, and the distance between the feeder is calculated by average distance

$$
L\left(K_{i}, K_{j}\right)=\frac{1}{n_{k i} n_{k j}} \sum \sum\left|N_{i}-N_{j}\right|
$$

where $K$ are the typical topologies, $N$ are the number of the feeders, $n_{k i}$ and $n_{k j}$ are respectively the number of samples in $K_{i}$ and $K_{j}$.

\section{ESTIMATION METHOD FOR NETWORK RELIABILITY}

In equation (1)-(2), how to calculate $\beta$ quickly is the core problem of the improved estimation algorithm. Therefore, this paper calculates the $\beta$ of the typical topology under different sections, and then obtain the expression of $\beta$ through regression analysis.

$$
\beta_{m k}=\sum_{j=1}^{N_{E k}} N_{m j} / \sum_{i=1}^{N_{D}} N_{i} N_{E k} \quad m=A, B, C
$$

where $N_{D}$ is the total number of feeder nodes, $N_{E k}$ is the total number of class $k$ devices, $N_{m j}$ is the number of class $m$, users after the failure of equipment $j$.

\section{A. Analysis of failure isolation capability}

The fault isolation capability is mainly reflected by $\beta_{A k}$ and it gets stronger as $\beta_{A k}$ increases. In practice, $0 \leq \beta \leq 1$ and the increased segments in feeder would lead to the more robust fault isolation capability. Therefore, the $\beta_{A k}$ will increase as the number of feeder segments $\left(P_{F}\right)$ increases until $\beta_{A k}$ reaches a certain limit in the region.

From Fig. $\mathrm{A}(a)$ in [2], the functional relationship between $\beta_{A k}$ and $P_{F}$ could be established by regression analysis.

$$
\begin{gathered}
f(x)=a e^{-b x}+c \\
f(x)=a(1+b / x)^{x} \\
f(x)=(a x+b) /(x+c)
\end{gathered}
$$

In the situation of switch failure, circuit breaker fails to isolate fault or transfer load. In this case, the change trend of $\beta_{A k}$ with $P_{F}$ is similar to the Sigmoid function from Fig. A $(b)$ in [2], the function is introduced into the regression analysis.

$$
f(x)=1 /\left(a+e^{-b x+c}\right)
$$

The constraint $0 \leq \lim f(x) \leq 1$ is applied to the regression function, the function with the highest correlation coefficient is taken as the formula of $\beta_{A k}$ and denote as $\beta_{A k}=f_{l}\left(P_{F}\right)$.

\section{B. Analysis of load transfer capability}

The tie line is added at end of the feeders, the users downstream of the fault section can be supplied by the backup power. After the failure of any devices in the feeder, only $\mathrm{A}, \mathrm{B}$ users exist, and $\beta_{A k}+\beta_{C k}=1$. Therefore, a similar regression method can be used to solve the $\beta_{C k}=f_{2}\left(P_{F}, P_{Z}\right)$. Meanwhile, a simplified method is adopted to reduce the complexity of $\beta_{C k}$. For each $P_{F}$, the regression effect of $\beta_{C k}$ on $P_{F}$ is solved under the condition of the supply capacity of the feeder $\left(P_{Z}=0 \%\right.$ and $\left.P_{Z}=100 \%\right)$. Ultimately, a regression linear relationship is used to represent the effect of $P_{Z}$ on $\beta_{C k}$.

\section{Analysis of other impact factors}

Distribution automation terminals can effectively identify the fault location and reduce fault clearance time. If the feeder contains such terminals, the $T_{d w}$ and $\beta$ will be rectified.

In case of the closed single-loop network with double power supply, the fault segments will be isolated by protection, which leaves the rest of the network structure still energized by power supply. Therefore, the $T_{d w}, T_{d n}, T_{g}$, and $T_{z}$ should be set to 0 in the calculation, which could improve the reliability of distribution network.

\section{Method Implementation}

Based on the above analysis, the pseudo-code for implement the proposed method is illustrated as follows.

Algorithm for Method Implementation

\begin{tabular}{cl}
\hline 1 & Initialization: $P_{F}, P_{Z}, N_{E}, u_{x}, P_{D E}, P_{D S}, T$, etc. \\
\hline 2 & Input the feeder topology structure \\
\hline 3 & Get the typical topology based on (5) \\
\hline 5 & for $i \leftarrow 1, \ldots, N$ \\
\hline 6 & for $j \longleftarrow 1, \ldots, N_{E}$ \\
\hline 7 & Deduce the $f_{1}, f_{2}$ based on (7)-(10) \\
\hline 8 & Set the $\lambda_{k}, T_{x}$ according to the types of blackout \\
\hline 9 & Modified $\beta, T_{d w}$ according to the number of terminals \\
\hline 10 & Modified $T_{d w}, T_{g}, T_{z}$ according to the mode of operation \\
\hline 11 & Calculate the (1) and (2) to obtain the reliability index \\
\hline
\end{tabular}




\section{CASE STUDY}

The real urban distribution network with specific topology [6] is selected to verify the feasibility and effectiveness of the proposed algorithm. The network includes $6 \times 110 \mathrm{kV}$ substations with a total capacity of 636MVA and $4 \times 35 \mathrm{kV}$ substations with a total capacity of 71MVA. Set the $C_{d}$ and $C_{i}$ as 1 while $C_{c}$ as 0 . After applying the TED based hierarchical clustering algorithm, the $96 \times 10 \mathrm{kV}$ feeders can be clustered into 4 types. The clustering results are shown in Fig 3.

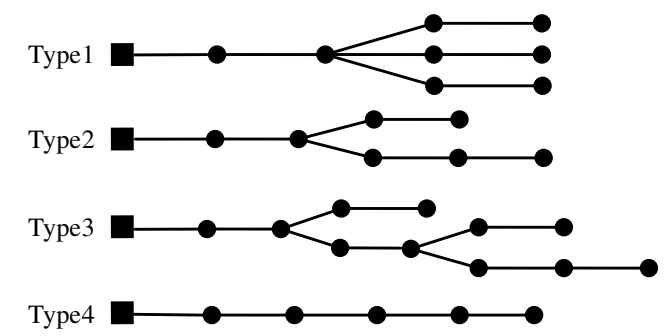

Fig. 3. Typical topology of each clustered feeder structure

It can be concluded that the proportions of each typical structure are $14 \%, 24 \%, 20 \%$, and $42 \%$ respectively. The 4 types are distinct in terms of network topology: Type 1 has multiple sub-branches, Type 2 has double sub-branches, Type 3 has multiple layers of sub-branches, while Type 4 has the single branch structure.

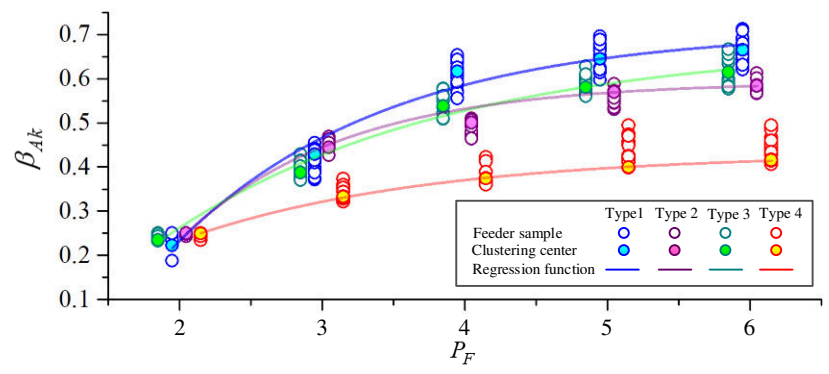

Fig. 4. Distribution of $\beta_{A k}$ in 4 types of clustered topology

As shown in Fig. 4, under the same $P_{F}\left(P_{F}>2\right)$, the $\beta_{A k}$ of type 1 is the largest, while the $\beta_{A k}$ of type 4 is the smallest. The $\beta_{A k}$ curves of types 2 and 3 are similar and located between types 1 and 4 . In addition, the $\beta_{A k}$ of the four topologies increase with the growth of the number of line segments $\left(P_{F}\right)$, and finally tends to flatten.

Under different types of faults, the fault isolation capability and load transfer capability of each typical topology are analyzed, and the corresponding regression function is calculated in Table I.

TABLE I

FUNCTION OF $\beta$ PARAMENTS

\begin{tabular}{c|c|c}
\hline \multirow{2}{*}{ Type } & \multicolumn{2}{|c}{ Regression Function } \\
\cline { 2 - 3 } & $\boldsymbol{\beta}_{\boldsymbol{A} \boldsymbol{k}\left(\boldsymbol{P}_{\mathbf{Z}}=\mathbf{0}\right)}$ & $\boldsymbol{\beta} \boldsymbol{C k}\left(\boldsymbol{P}_{\mathbf{Z}=\mathbf{1 0 0 \%})}\right.$ \\
\hline 1 & $0.7 e^{-0.25 P_{F}}+0.77$ & $0.57 e^{-0.14 P_{F}}+0.045$ \\
\hline 2 & $\left(0.73 P_{F}-2.03\right) /\left(P_{F}-1.8\right)$ & $\left(0.19 P_{F}-0.25\right) /\left(P_{F}-3.36\right)$ \\
\hline 3 & $\left(0.68 P_{F}-2.9\right) /\left(P_{F}-3.88\right)$ & $\left(0.22 P_{F}-0.54\right) /\left(P_{F}-3.9\right)$ \\
\hline 4 & $\left(0.5 P_{F}-1.0\right) /\left(P_{F}-1.01\right)$ & $\left(0.5 P_{F}-0.013\right) /\left(P_{F}-1.0\right)$ \\
\hline
\end{tabular}

The reliability index can be calculated through regression function obtained from Table I. The calculation error of the proposed algorithm is solved by network equivalent method and compare with approximate estimation algorithm.

TABLE II

COMPARISON OF CALCULATION ERRORS UNDER RELIABILITY INDEX

\begin{tabular}{c|c|c}
\hline \multirow{2}{*}{ Type } & \multicolumn{2}{|c}{ Average Errors of SAIDI } \\
\cline { 2 - 3 } & Reference [4] & Proposed Algorithm \\
\hline 1 & $21.32 \%$ & $6.13 \%$ \\
\hline 2 & $15.74 \%$ & $4.06 \%$ \\
\hline 3 & $16.89 \%$ & $5.75 \%$ \\
\hline 4 & $3.12 \%$ & $3.06 \%$ \\
\hline total & $11.26 \%$ & $4.16 \%$ \\
\hline
\end{tabular}

As shown in Table II, the computational error of the proposed algorithm is reduced by $7.74 \%$ compared with that by the algorithm in [4]. For type 4, the two algorithms have similar errors due to the simplicity of the network topology. The reliability of other feeders can be estimated by using the parameters of the typical topology.

The proposed reliability estimation method can help improve the solving efficiency, particularly applied in the optimal distribution network planning, and an extended case is given at Section B in [2].

\section{CONCLUSION}

This paper presents an improved method for estimating distribution network reliability. TED based hierarchical clustering method is used to identify the typical topology structure of feeders in the area to be evaluated, and cluster the similarity feeders for network reliability assessment. In addition, the proposed method could deduce the formula of reliability index and the corresponding $\beta$ through regression analysis. Case study shows its effectiveness and advantages in network reliability evaluation and planning.

\section{REFERENCES}

[1] IEEE PES Society, IEEE tutorial on electric delivery system reliability evaluation. Montreal: IEEE, May. 2006.

[2] [Online]. Available: https://github.com/eexxyy/Relibility/blob/master/Appendix.pdf

[3] Y. Liu, Y. Su, Y. Xiang, et. al, "Operational reliability assessment for gas-electric integrated distribution feeders," IEEE Trans. Smart Grid., vol. 10, pp.1091-1100, Jan. 2019.

[4] L. Guan, Y. Feng, S. Liu, et al. "Approximate evaluation algorithm for reliability indices of cosmically distribution system," Proceedings of the CSEE, vol. 26, no. 10, pp. 92-98, 2006.

[5] K. C. Tai, "The tree-to-tree correction problem," J. ACM, vol. 26, no. 3, pp. 422-433, 1979.

[6] Y. Su, J. Liu, Y. Liu, et al. "Optimization model of selecting power supply reliability reconstruction measures in large-scale MV distribution network and its solution method," Power System Technology, vol. 41, no. 1, pp. 212-221, Jan. 2017. 